\title{
Prognostic implications of epicardial fat volume quantification in acute pericarditis
}

\author{
George Lazaros*,a , Alexios S. Antonopoulos ${ }^{*}, t, a$, Evangelos K. Oikonomou ${ }^{*}{ }^{\dagger}$, Panagiotis Vasileiou* \\ Evangelos Oikonomou ${ }^{*}$, Evangelia Stroumpouli ${ }^{\ddagger}$, Apostolos Karavidas $^{*}$, Charalambos Antoniades $^{\dagger}$ and \\ Dimitris Tousoulis* \\ *1st Cardiology Department, Hippokration Hospital, Athens Medical School, Athens, Greece, ${ }^{\dagger}$ Division of Cardiovascular \\ Medicine, University of Oxford, Oxford, UK, ' Radiology Department, Hippokration Hospital, Athens, Greece
}

\begin{abstract}
Background The pathophysiology of acute pericarditis remains largely unknown, and biomarkers are needed to identify patients susceptible to complications. As adipose tissue has a pivotal role in cardiovascular disease pathogenesis, we hypothesized that quantification of epicardial fat volume (EFV) provides prognostic information in patients with acute pericarditis.

Materials and methods Fifty $(n=50)$ patients with first diagnosis of acute pericarditis were enrolled in this study. Patients underwent a cardiac computerized tomography (CT) scan to quantify EFV on a dedicated workstation. Patients were followed up in hospital for atrial fibrillation (AF) development and up to 18 months for the composite clinical endpoint of development of constrictive, recurrent or incessant pericarditis or poor response to nonsteroidal anti-inflammatory drugs.
\end{abstract}

Results Patients presenting with chest pain had lower EFV vs. patients without chest pain (167.2 \pm 21.7 vs. $105.1 \pm 11.1 \mathrm{~cm}^{3}$, respectively, $P<0.01$ ); EFV (but not body mass index) was strongly positively correlated with pericardial effusion size $(r=0.395, P=0.007)$ and associated with in-hospital AF. At follow-up, patients that reached the composite clinical endpoint had lower EFV $(P<0.05)$. After adjustment for age, EFV was associated with lower odds ratio for the composite clinical endpoint point of poor response to NSAIDs or the development of constrictive, recurrent or incessant pericarditis during follow-up (per $20 \mathrm{~cm}^{3}$ increase in EFV: $\mathrm{OR}=0.802$ [0.656-0.981], $P<0.05)$.

Conclusions We report for the first time a significant association of EFV with the clinical features and the outcome of patients with acute pericarditis. Measurement of EFV by CT may have important prognostic implications in these patients.

Keywords Acute pericarditis, adipose tissue, computer tomography imaging, epicardial fat.

Eur J Clin Invest 2017; 47 (2): 129-136

Acute pericarditis is a relatively common disorder with a reported incidence of 27.7 cases per 100000 persons per year in the Western world [1,2]. Potential complications of acute pericarditis include pericarditis recurrence, cardiac tamponade and constrictive pericarditis [3]. To date, there is a limited clinical evidence on prognostic factors of outcome in acute pericarditis; corticosteroid therapy, nonuse of colchicine, persistent C-reactive protein elevation (CRP) beyond the first week of treatment and the underlying aetiology have been previously associated with the recurrence of acute pericarditis [4-6].

Recent evidence suggests that epicardial fat affects cardiac biology via the release of adipokines in a paracrine or endocrine

\footnotetext{
${ }^{a}$ Authors equally contributed.
}

manner [7]. Epicardial fat could also have a role in the pathophysiology of acute pericarditis (given its close anatomical affinity with the pericardium), although this hypothesis has never been explored.

Study of epicardial fat biology is not easily accessible in humans, but indirect information can be derived from quantification of epicardial fat volume (EFV) by the use of computerized tomography (CT) imaging; EFV offers independent prognostic information on the outcome of subjects undergoing noncontrast CT for coronary calcium scoring [8,9], but its prognostic value in patients with acute pericarditis is unknown. In a published case report, excessive epicardial adiposity has been associated with constrictive pericarditis [10], but there has been no systematic investigation of the 
relationship between EFV and the clinical course of acute pericarditis.

We hypothesized that EFV quantification is associated with the clinical features of acute pericarditis at presentation and offers prognostic information on the clinical outcome of these patients. We designed a clinical study to investigate the value of quantifying EFV as a biomarker in patients presenting with a first-episode acute pericarditis.

\section{Methods}

\section{Study population}

We prospectively screened a total of 135 patients with pericarditis admitted to the Center for the Diagnosis and Treatment of Pericardial Diseases, 1st Cardiology Department, Hippokration Hospital, Athens, Greece, between January 2009 and July 2013. Patients were enrolled in the study if they were admitted for a first episode of acute pericarditis. Exclusion criteria were known history of recurrent pericarditis, myopericarditis, previous anti-inflammatory treatment for the index episode in an outpatient setting and CT scan performed later than $48 \mathrm{~h}$ from hospital admission. As our Hospital is a referral centre for pericarditis in Greece, the majority of the patients were referrals that had already received treatment for the index episode or complicated cases of pericarditis. Therefore, out of the 135 screened subjects, a total of 50 consecutive patients were eligible and included in the study (population demographics, Table 1). Written informed consent was obtained from all participants, and the protocol was approved by the Institutional Ethics Committee (ref. number 14167/20/08/ 2009) and carried out in accordance with the principles of the Declaration of Helsinki.

\section{Diagnosis of acute pericarditis and choice of treatment}

Acute pericarditis was diagnosed based on current clinical recommendations at the time of enrolment [11]. Baseline clinical and demographic data were recorded in all cases. In line with the current guidelines, diagnostic work-up included clinical history and examination, ECG, chest X-ray, echocardiography and routine blood tests, including among others high-sensitivity cardiac troponin I and high-sensitivity CRP (hsCRP). In patients with high-risk features for secondary forms or a complicated course of acute pericarditis [12], additional tests were performed at the discretion of the attending physician.

Ibuprofen 1,8gr daily or aspirin 3gr/day was given as firstline therapy for at least 7-10 days, provided that hsCRP was normalized within the same time interval, and subsequent tapering in the following 3-4 weeks was accomplished.
Table 1 Demographics of the study population

\begin{tabular}{|c|c|}
\hline Participants $(n)$ & 50 \\
\hline Age (years) & $61 \cdot 7 \pm 2 \cdot 6$ \\
\hline Male gender $(\%)$ & $30(60)$ \\
\hline Smoking: active-ex (\%) & $12(24)$ \\
\hline Arterial hypertension (\%) & $25(50)$ \\
\hline Dyslipidaemia (\%) & $10(20)$ \\
\hline Diabetes mellitus (\%) & $22(44)$ \\
\hline Systolic BP (mmHg) & $123 \cdot 3 \pm 2 \cdot 6$ \\
\hline Diastolic BP (mmHg) & $77.4 \pm 1.5$ \\
\hline Body mass index $\left(\mathrm{kg} / \mathrm{m}^{2}\right)$ & $27.45 \pm 0.81$ \\
\hline Chest pain (\%) & $34(68)$ \\
\hline Fever (\%) & 19 (38) \\
\hline Cough (\%) & $10(20)$ \\
\hline Dyspnoea (\%) & $30(60)$ \\
\hline ST elevation (\%) & $6(12)$ \\
\hline PR depression (\%) & $5(10)$ \\
\hline T wave abnormalities (\%) & $28(56)$ \\
\hline Electrical alternans (\%) & $0(0)$ \\
\hline $\begin{array}{l}\text { Sinus rhythm/chronic atrial } \\
\text { fibrillation }(\%)\end{array}$ & $34(68) / 9(18)$ \\
\hline New onset atrial fibrillation (\%) & $7(14)$ \\
\hline Tamponade at presentation (\%) & $0(0)$ \\
\hline Pericardial effusion (\%) & $41(82)$ \\
\hline $\operatorname{WCC}(n / \mu \mathrm{L})$ & $11374 \pm 1025$ \\
\hline Polymorphonuclear (\%) & $73 \cdot 3 \pm 1 \cdot 6$ \\
\hline CRP at admission (mg/L) & $65.9[23 \cdot 5-139.7]$ \\
\hline $\mathrm{ESR}(\mathrm{mm})$ & $70 \cdot 6 \pm 8.6$ \\
\hline
\end{tabular}

CRP, C-reactive protein; BP, blood pressure; ESR, erythrocyte sedimentation rate; WCC, white cell count.

Steroids (prednisolone $0 \cdot 2-0.5 \mathrm{mg} / \mathrm{kg} /$ day or other steroid equivalent dose) were given until hsCRP normalization and symptoms' resolution followed by dose tapering [11]. Steroids were reserved for secondary causes of pericarditis requiring such treatment and contraindications or poor response to NSAIDs treatment (persisting symptoms and/or hsCRP elevation beyond 10 days of treatment). Colchicine $1 \mathrm{mg} /$ day (with dose adjustment when appropriate) was given in all cases unless contraindicated. Treatment failure with first-line medication and/or switch to steroids for any reason was recorded. 


\section{Clinical follow-up}

All patients were followed up in hospital, for the development of atrial fibrillation (AF), defined as 'a first diagnosed episode of AF lasting for at least $30 \mathrm{~s}$. All patients were advised to report any abnormality or irregularity in the heart rhythm. AF episodes were detected by either continuous ECG monitoring or 12-lead ECG if palpitations were reported. Moreover, in an individualized basis Holter monitoring was adopted to unveil episodes of AF.

All participants were also followed up at outpatients' clinic at 2 weeks, 1 month and 3 months posthospital discharge and every 3 months thereafter (or earlier if symptomatic) up to a total of 18 months, given that the majority of clinical complications appear within this time frame [13]. The composite clinical endpoint was poor response to NSAIDs treatment (defined by the need to switch to corticosteroid therapy) or the development of incessant, recurrent or constrictive pericarditis. Pericarditis was defined as incessant in case of symptoms recurrence either within 6 weeks after drug discontinuation or during attempted weaning and as recurrent in cases of symptoms reappearance beyond 6 weeks of treatment withdrawal [14]. The diagnosis of constrictive pericarditis was established in the presence of symptoms and signs of right heart failure due to impaired diastolic filling, as unveiled by a combination of the available imaging.

\section{Quantification of Epicardial fat volume by CT imaging}

CT imaging was performed in all cases within $48 \mathrm{~h}$ from hospital admission as a part of this study protocol. Patients underwent a noncontrast cardiac CT scan on a 16-slice CT scanner (Toshiba Activion $16^{\mathrm{TM}}$, Tokyo, Japan). A noncontrast axial acquisition CT scan was obtained using the established institutional CT scan protocol (1 mm axial slice thickness, tube energy of $120 \mathrm{keV}$ and $\mathrm{mA}$ automatically adjusted for body size), covering the entire cardiac silhouette. The reconstructed images were transferred to an offline image processing and analysis workstation (Aquarius Workstation ${ }^{\circledR}$ V.4.4.11, TeraRecon, Inc., Foster City, CA, USA). Adipose tissue was defined as all voxels with attenuation between -190 and -30 Hounsfield Units (HU) as previously suggested [15]. EFV was tracked by contouring of the pericardial sac in a semi-automated method (starting cranially from the bifurcation of the pulmonary artery and extending caudally up to the apex of the heart).

Technical considerations: The presence of pericardial effusion may lead to technical difficulties in the measurement of EFV. However, as any pericardial effusion has a CT attenuation equal to or above water $(>0 \mathrm{HU})$, there was no overlapping between pericardial effusion and epicardial fat which has completely different CT attenuation thresholds (between -190 and $-30 \mathrm{HU})$. A representative example of EFV quantification by CT in a patient with acute pericarditis with pericardial effusion is demonstrated in Fig. 1.

\section{Pericardial effusion quantification}

A semi-quantitative echocardiographic assessment was used for the assessment of pericardial effusion size, which was accordingly classified as mild when its maximal diameter in diastole measured less than $10 \mathrm{~mm}$ in all pericardial spaces, moderate if between 10 and $20 \mathrm{~mm}$ and large if $>20 \mathrm{~mm} \mathrm{[16].}$

\section{Statistical analysis}

Non-normally distributed variables are presented as median [25th-75th percentile]. Normally distributed variables are presented as mean \pm standard error of mean (SEM). Comparisons of characteristics between different groups of patients were performed using unpaired t-test for two groups or ANOVA as appropriate. Non-normally distributed continuous variables were compared between patient subgroups by the use of Mann-Whitney U-test. Categorical variables were compared by using chi-squared test, as appropriate. Correlations between continuous variables were assessed by using bivariate analysis, and Pearson's $r$ or Spearman's rho coefficient was estimated as appropriate. To test whether EFV independently predicts atrial fibrillation or the composite clinical endpoint, a binary logistic regression model was used with independent variables those that showed correlation $P<0.10$ with the dependent variable in bivariate analysis. The results of the regression analysis are presented as standardized beta [ $95 \%$ confidence intervals] and $P$ values. The discriminative value of EFV was explored in receiver operating characteristic (ROC) curve analysis by using the binary variable, that is $\mathrm{AF}$, as the outcome of interest and EFV as a continuous test variable; the discriminative value of the test variable was measured by calculating the area under the curve (AUC). For power calculations, we were based on previous clinical experience from our centre (estimating that approximately $55 \%$ of patients with acute pericarditis have a complicated disease course) and available pilot data on epicardial fat volume quantification. A priori power calculations estimated that with a study population of 49 subjects we would be able to detect a 50\% difference in EFV (assuming a standard deviation of 38 for EFV) between subjects with a benign disease course compared to patients that reached the composite clinical endpoint, with power 0.90 and $\alpha=0.05$.

\section{Results}

\section{Epicardial fat volume and clinical presentation of acute pericarditis}

We first explored the association of EFV with clinical and echocardiographic features of acute pericarditis at presentation. 

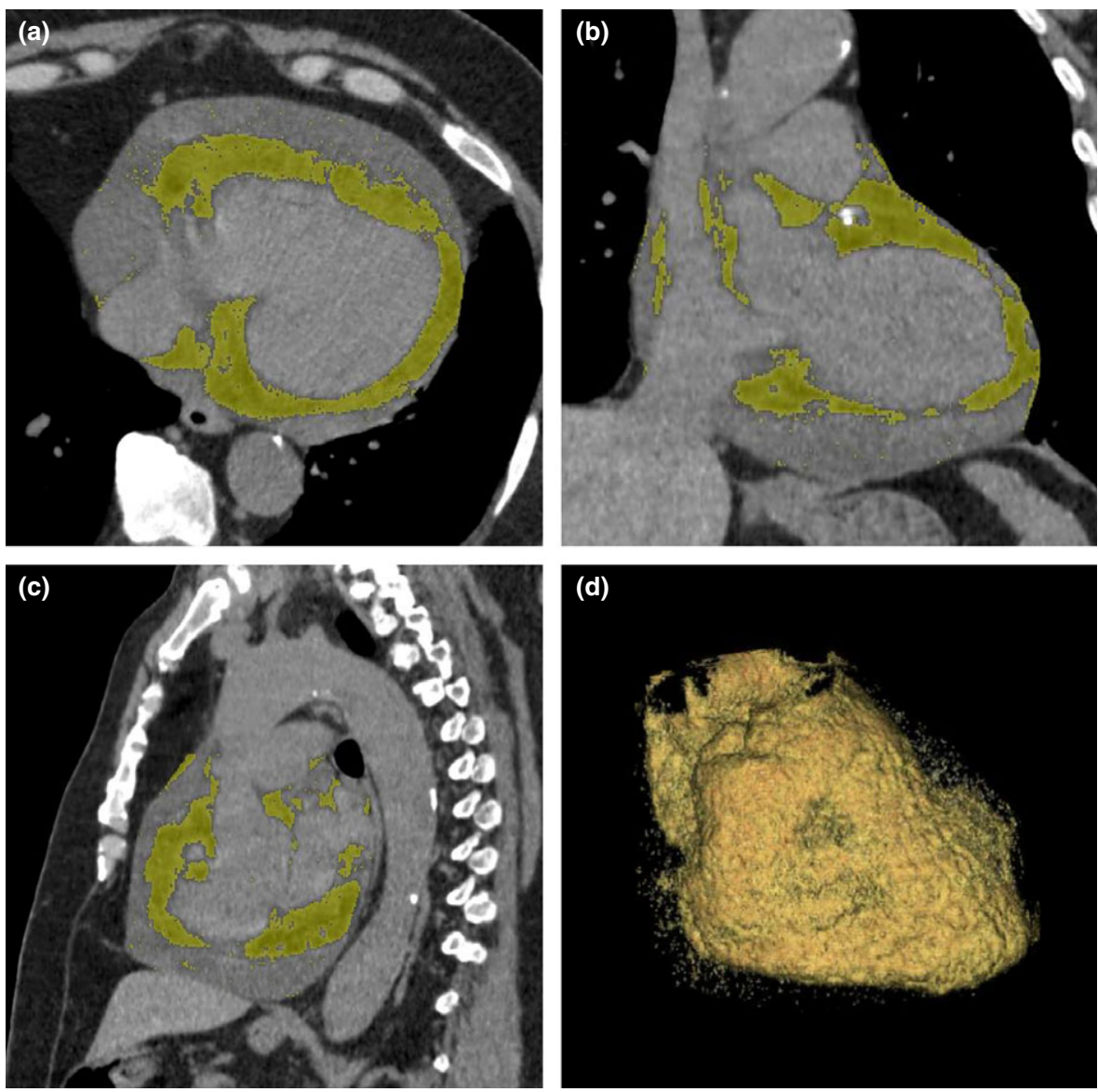

Figure 1 Representative example of epicardial fat volume quantification by computerized tomography imaging in acute pericarditis with pericardial effusion; fat is highlighted in yellow, a: axial, b. coronal, c: sagittal view and d. volumerendered reconstruction of tracked epicardial fat.
In bivariate analysis, EFV was not associated with clinical (dyspnoea, fever) or electrocardiographic features of acute pericarditis at presentation (PR depression, ST elevation, T wave abnormalities or electrical alternans, $P=\mathrm{NS}$ for all). Interestingly though, patients with chest pain had higher EFV (Fig. 2a), but not body mass index. We then searched for other confounders that could explain this association, such as the presence of pericardial effusion; 41 of 50 patients (82\%) had pericardial effusion at admission, and its size was negatively associated with chest pain at admission $(\rho=-0.438$,

$P<0.0001)$. Pericardial effusion size was also positively associated with EFV (Fig. 2b), which could also explain the association of the latter with chest pain at presentation. There was no association between pericardial effusion size and obesity as assessed by BMI ( $P=$ NS, not shown). This observation implied that epicardial fat may be directly implicated in the mechanisms of pericardial effusion development in acute pericarditis.

\section{Epicardial fat volume and systemic inflammation/ myocardial injury}

There was a trend towards an inverse association of EFV with $\log ($ hsCRP) levels at admission $(r=-0 \cdot 247, P=0 \cdot 10)$ which did not reach, however, statistical significance.

BMI was not associated with hsCRP admission levels, while there was also no association of either EFV or BMI with admission or maximum cardiac troponin I levels $(P=$ NS for both).

\section{Epicardial fat volume and atrial fibrillation development}

At enrolment, patients were either at sinus rhythm $(n=41)$ or at atrial fibrillation $(n=9)$. No other arrhythmias were observed. After excluding those patients with chronic atrial fibrillation ( $\mathrm{AF}, n=9$ ), we observed a significant interaction between the development of new paroxysmal AF and BMI. Size of pericardial effusion was not associated with new AF development (Fig. 2c), but patients that developed new AF during hospitalization $(n=7)$ had higher BMI (Fig. 2d) and higher EFV (Fig. 2e) compared to patients that maintained sinus rhythm. In ROC curve analysis, EFV measurement had good accuracy for the development of new AF (AUC = 0.828, $P=0.012$ ); for a cut-off value of $123.5 \mathrm{~cm}^{3}$, EFV had $83.3 \%$ sensitivity and $71.9 \%$ specificity for detecting those patients that developed new AF. 
Figure 2 Higher epicardial fat volume (EFV) was associated with chest pain symptoms (a, patients with chest pain, $n=34)$ and the size of pericardial effusion (b, no/small: $n=12$, medium: $n=16$, large: $n=22$ ) in patients with acute pericarditis. The size of pericardial effusion was not associated with the development of paroxysmal atrial fibrillation $(\mathrm{pAF}, \mathrm{c})$. Both body mass index (BMI, d) and EFV (e) were significantly higher in patients that developed new pAF $(n=7)$ compared to those maintaining sinus rhythm (SR, $n=34)$; patients with known $\operatorname{AF}(n=9)$ were excluded from the analysis. EFV was significantly lower in patients diagnosed with a secondary (2ndary) cause of pericarditis ( $n=16, f)$. Similarly, EFV was significantly lower in patients that required switch to second-line therapy $(n=15, \mathrm{~g})$ and those that had a more severe disease course as defined by the composite clinical endpoint of the development of incessant, recurrent or constrictive, recurrent or incessant pericarditis and/or need for treatment up-titration ( $n=28, \mathrm{~h})$. complic. = complicated.
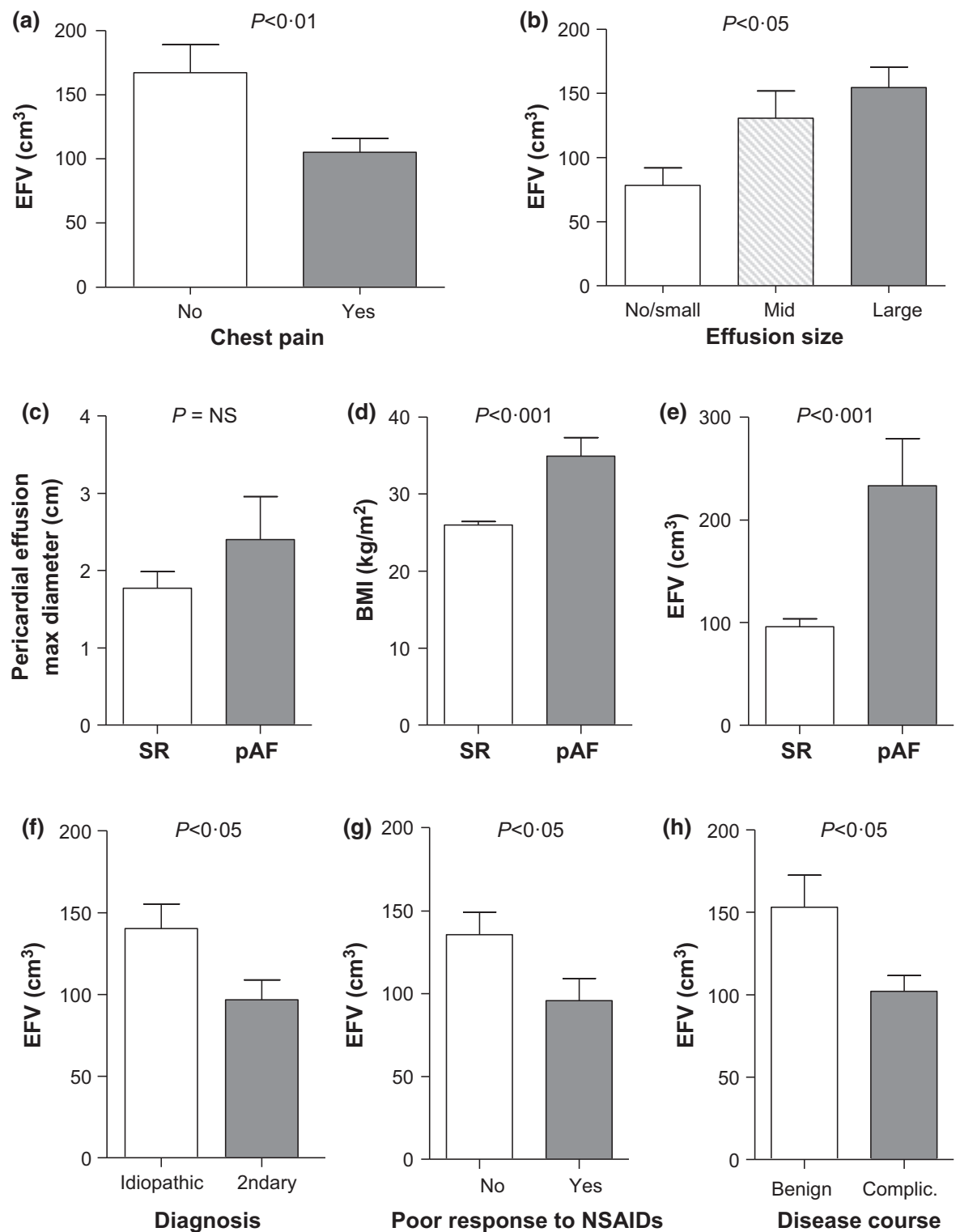

\section{Epicardial fat volume, diagnostic and therapeutic implications}

Interestingly, patients with acute pericarditis secondary to other causes ( $n=16$ or $32 \%$ of the cohort) had lower EFV compared to patients with idiopathic pericarditis (Fig. 2f). During clinical follow-up, a total of 28 patients (56\%) reached the composite clinical endpoint of development of incessant, recurrent or constrictive pericarditis or poor response to NSAIDs; in more details, 19 (38\%) patients developed incessant/recurrent pericarditis, four $(8 \%)$ patients developed constrictive pericarditis and seven patients $(14 \%)$ had a poor response to NSAIDs. In search of predictors of a poor response to first-line therapy, we observed that poor responders to NSAIDs had lower EFV (Fig. 2g), while there was no difference in BMI between the two groups (not shown, $P=\mathrm{NS}$ ). Moreover, patients with lower EFV had also a poorer disease course as defined by the composite clinical endpoint (Fig. 2h). In logistic regression analysis, EFV remained (after adjustment for age) an independent risk factor for the composite clinical endpoint (Table 2) in this cohort of 50 patients with first diagnosis of acute pericarditis. For every $20 \mathrm{~cm}^{3}$ increase in EFV, there was an odds ratio of 0.802 [95\% CI: 0.656-0.981, $P<0.05$ ] for the composite endpoint of poor response to NSAIDs therapy or the development of incessant, recurrent or constrictive pericarditis. 
Table 2 Multivariable logistic regression analysis for the composite clinical endpoint predictors of poor response to NSAIDs therapy or the development of incessant, recurrent or constrictive pericarditis

\begin{tabular}{|c|c|c|c|}
\hline \multicolumn{4}{|l|}{ Univariate analysis } \\
\hline Variable & \multicolumn{2}{|c|}{ Correl.coefficient } & $P$-value \\
\hline Age & \multicolumn{2}{|l|}{-0.174} & 0.045 \\
\hline Epicardial fat volume ${ }^{\dagger}$ & \multicolumn{2}{|l|}{-0.266} & 0.078 \\
\hline \multicolumn{4}{|c|}{ Multivariable analysis ( $R^{2}$ for the model: $\left.0.162, P<0.0001\right)$} \\
\hline Variable & Correl.coefficient & $\begin{array}{l}\text { Odds ratio } \\
{[95 \% \mathrm{Cl}]}\end{array}$ & $P$-value \\
\hline Epicardial fat volume & -0.220 & $\begin{array}{l}0.802 \\
{[0.656-0.981]}\end{array}$ & 0.032 \\
\hline
\end{tabular}

${ }^{\dagger}$ Reported in increments of $20 \mathrm{~cm}^{3}$.

\section{Discussion}

In the present paper, we report for the first time an association between EFV and the clinical presentation and disease course of patients with acute pericarditis. In patients with a first episode of acute pericarditis, we demonstrated that EFV as measured by CT imaging measurement of EFV may have important prognostic implications. Patients with lower EFV present less frequently with chest pain and are at increased risk for a more severe disease course at long term, as defined by the composite endpoint of poor response to NSAIDs treatment or the development of incessant, recurrent or constrictive pericarditis.

Acute pericarditis is an inflammatory pericardial syndrome, but its exact pathophysiology or the mechanisms regulating pericardial effusion accumulation have not been fully clarified [14]. In this study, we report a significant positive association between the size of pericardial effusion and EFV. We have recently demonstrated that human adipose tissue communicates with the cardiovascular system [17] and highlighted the importance of local fat depots such as epicardial [7] or perivascular $[18,19]$ fat in regulating the function of the heart/ vessels, respectively. Similarly, the inflammatory status and the vascular permeability of pericardium could be affected by adipokines released by epicardial fat, but this hypothesis has never been explored. Indeed, adipokines have been identified in human pericardial fluid analysis [20], but their biological significance remains unknown. Our findings provide the first clinical evidence on a relationship between epicardial fat and pericardial effusion development in acute pericarditis and suggest a possible involvement of epicardial fat in the pathophysiology of acute pericarditis.
Development of new AF is a known complication in acute pericarditis [21]. In our study, we demonstrate that patients with higher EFV were at increased risk for new AF development during a first episode of acute pericarditis, a finding which is expected and in agreement with previous robust clinical evidence suggesting that increased EFV is a risk factor for AF $[22,23]$.

AF occurrence may increase the risk for future AF events, but does affect the long-term risk for recurrent or constrictive pericarditis [1], which are the main complications after a first episode of acute pericarditis. Colchicine halves risk of recurrences [24], while steroids promote recurrences possibly due to their negative effect on the host's defensive mechanisms. Pericardial effusion size at presentation does not offer prognostic information on the risk of recurrences, but other factors such as a secondary cause of pericarditis [5], incomplete response to anti-inflammatory therapy with persistent hsCRP at week 1 [5], interleukin-8 elevation [25] and MHC class I chain-related protein A [26] have been related to poorer prognosis. Nevertheless, the identification of acute pericarditis patients at risk for a poorer disease course remains a challenging issue.

In our study, we demonstrate that increased EFV is associated with better long-term clinical outcome. The interplay of obesity and adipose tissue with the cardiovascular system is indeed a complex one $[7,17,27]$. In clinical studies, higher EFV has been associated with an increased risk for future cardiovascular events in CAD patients, while on the other hand, reduced EFV flags a more advanced disease state in HF patients [27]. Thus, the role of EFV as a cardiovascular biomarker seems to be modulated by the underlying disease condition, as we have previously shown epicardial fat changes its biology in response to signals that it receives from the heart [7]. It is possible that lower EFV in patients with pericarditis could be the result of local inflammatory activation, inducing adipose tissue de-differentiation and lipolysis [17], and reflect another aspect of the 'obesity paradox', that is the better clinical outcome of obese patients with established cardiovascular disease [27]. The association between EFV and long-term clinical outcome is an important one as the risk of recurrent, incessant or constrictive pericarditis and/or need for treatment up-titration have a major impact on the quality of life and prognosis of these patients, and biomarkers able to predict these events could be of value in patients presenting with acute pericarditis.

It should be noted that our study enrolled only patients with a first episode of acute pericarditis before initiation of any treatment; patients presenting with a pericarditis recurrence, myopericarditis or already on treatment for the indexed episode were excluded from the study. Therefore, the exclusion criteria used as well as the small-study population limit the extrapolation of our observations to all patients with pericarditis (e.g. complicated cases). 
Nevertheless, our findings support the notion that epicardial fat is involved in the pathophysiology of acute pericarditis. $\mathrm{EFV}$, as quantified by CT imaging, is independently associated with the clinical outcome of patients with a first episode of acute pericarditis. If confirmed in larger clinical studies, our findings may have important prognostic implications for the management of patients with acute pericarditis. Moreover, the higher rate of NSAIDs failure linked to lower EFV may have therapeutic implications for these patients, namely a lower threshold for switching to steroid administration. These findings provide new insights into the mechanisms involved in pericarditis syndrome and merit further investigation.

\section{Acknowledgements}

GL contributed to clinical data collection and to the writing of the manuscript, and is the guarantor of the paper, taking responsibility for the integrity of the work as a whole, from inception to published article; ASA conceived the study, participated in image analysis, wrote the manuscript and performed statistical analysis; EKO contributed to image data analysis, and PV/EO/ES/AK contributed to clinical data collection; CA and DT reviewed the manuscript.

\section{Declarations of interest and funding information}

The authors have nothing to declare.

\section{Address}

1st Cardiology Department, Hippokration Hospital, Athens Medical School, Vas. Sofias Avenue 11411528 Athens, Greece (G. Lazaros, A. S. Antonopoulos, E. K. Oikonomou, P. Vasileiou, E. Oikonomou, A. Karavidas, D. Tousoulis); Division of Cardiovascular Medicine, University of Oxford, Level 5, West Wing, Headley Way OX3 9DU, Oxford, UK (A. S. Antonopoulos, E. K. Oikonomou, C. Antoniades); Radiology Department, Hippokration Hospital, Vas. Sofias Avenue 11411528 Greece (E. Stroumpouli).

Correspondence to: Dr Alexios S. Antonopoulos, Cardiology Department, Hippokration Hospital, Athens Medical School, Vas. Sofias 114, 11528, Athens, Greece. Tel.: +316947607442; fax: +3102132088026; e-mail: alexios.antonopoulos@car-

diov.ox.ac.uk

Received 4 October 2016; accepted 5 December 2016

\section{References}

1 Imazio M, Cecchi E, Demichelis B, Chinaglia A, Ierna S, Demarie D et al. Myopericarditis versus viral or idiopathic acute pericarditis. Heart 2008;94:498-501.

2 Lazaros G, Imazio M, Tousoulis D. Letter by Lazaros et al. Regarding Article, “Clinical Profile and Influences on Outcomes in
Patients Hospitalized for Acute Pericarditis". Circulation 2015;132: e127.

3 LeWinter MM. Clinical practice. Acute pericarditis. N EngL J Med 2014;371:2410-6.

4 Imazio M, Brucato A, Maestroni S, Cumetti D, Belli R, Trinchero R et al. Risk of constrictive pericarditis after acute pericarditis. Circulation 2011;124:1270-5.

5 Imazio M, Brucato A, Maestroni S, Cumetti D, Dominelli A, Natale $\mathrm{G}$ et al. Prevalence of $\mathrm{C}$-reactive protein elevation and time course of normalization in acute pericarditis: implications for the diagnosis, therapy, and prognosis of pericarditis. Circulation 2011;123:1092-7.

6 Imazio M, Spodick DH, Brucato A, Trinchero R, Adler Y. Controversial issues in the management of pericardial diseases. Circulation 2010;121:916-28.

7 Antonopoulos AS, Margaritis M, Verheule S, Recalde A, Sanna F, Herdman L et al. Mutual regulation of epicardial adipose tissue and myocardial redox state by PPAR-gamma/Adiponectin Signalling. Circ Res 2016;118:842-55.

8 Nakanishi R, Rajani R, Cheng VY, Gransar H, Nakazato R, Shmilovich $\mathrm{H}$ et al. Increase in epicardial fat volume is associated with greater coronary artery calcification progression in subjects at intermediate risk by coronary calcium score: a serial study using non-contrast cardiac CT. Atherosclerosis 2011;218:363-8.

9 Spearman JV, Renker M, Schoepf UJ, Krazinski AW, Herbert TL, De Cecco CN et al. Prognostic value of epicardial fat volume measurements by computed tomography: a systematic review of the literature. Eur Radiol 2015;25:3372-81.

10 Do GW, Ku BS, Park CS, Kim SJ, Shin ES, Choi SH et al. A case of constrictive pericarditis associated with huge epicardial fat volume. Korean Circ J 2009;39:116-20.

11 Maisch B, Seferovic PM, Ristic AD, Erbel R, Rienmuller R, Adler Y et al. Guidelines on the diagnosis and management of pericardial diseases executive summary; The Task force on the diagnosis and management of pericardial diseases of the European society of cardiology. Eur Heart J 2004;25:587-610.

12 Imazio M, Cecchi E, Demichelis B, Ierna S, Demarie D, Ghisio A et al. Indicators of poor prognosis of acute pericarditis. Circulation 2007;115:2739-44.

13 Imazio M, Demichelis B, Parrini I, Cecchi E, Demarie D, Ghisio A et al. Management, risk factors, and outcomes in recurrent pericarditis. Am J Cardiol 2005;96:736-9.

14 Lazaros G, Vlachopoulos C, Stefanadis C. Idiopathic recurrent pericarditis: searching for Ariadne's thread. Hellenic J Cardiol 2009;50:345-51.

15 Cheng VY, Dey D, Tamarappoo B, Nakazato R, Gransar H, MirandaPeats R et al. Pericardial fat burden on ECG-gated noncontrast CT in asymptomatic patients who subsequently experience adverse cardiovascular events. JACC Cardiovasc Imaging 2010;3:352-60.

16 Imazio M, Adler Y. Management of pericardial effusion. Eur Heart J 2013;34:1186-97.

17 Antonopoulos AS, Margaritis M, Coutinho P, Digby J, Patel R, Psarros C et al. Reciprocal effects of systemic inflammation and brain natriuretic peptide on adiponectin biosynthesis in adipose tissue of patients with ischemic heart disease. Arterioscler Thromb Vasc Biol 2014;34:2151-9.

18 Antonopoulos AS, Margaritis M, Coutinho P, Shirodaria C, Psarros C, Herdman L et al. Adiponectin as a link between type 2 diabetes and vascular NADPH oxidase activity in the human arterial wall: the regulatory role of perivascular adipose tissue. Diabetes 2015;64:2207-19. 
19 Margaritis M, Antonopoulos AS, Digby J, Lee R, Reilly S, Coutinho P et al. Interactions between vascular wall and perivascular adipose tissue reveal novel roles for adiponectin in the regulation of endothelial nitric oxide synthase function in human vessels. Circulation 2013;127:2209-21.

20 Yavuz S, Kasap M, Akpinar G, Ozbudak E, Ural D, Berki T. Analysis of pericardial effusion from idiopathic pericarditis patients by twodimensional gel electrophoresis. BioMed Res Int 2014;2014:942718.

21 Imazio M, Lazaros G, Picardi E, Vasileiou P, Orlando F, Carraro M et al. Incidence and prognostic significance of new onset atrial fibrillation/flutter in acute pericarditis. Heart 2015;101:1463-7.

22 Wong CX, Ganesan AN, Selvanayagam JB. Epicardial fat and atrial fibrillation: current evidence, potential mechanisms, clinical implications, and future directions. Eur Heart J 2016;doi: 10.1093/ eurheartj/ehw045.

23 Zhu W, Zhang H, Guo L, Hong K. Relationship between epicardial adipose tissue volume and atrial fibrillation: a systematic review and meta-analysis. Herz 2016;41:421-7.
24 Imazio M, Belli R, Brucato A, Cemin R, Ferrua S, Beqaraj F et al. Efficacy and safety of colchicine for treatment of multiple recurrences of pericarditis (CORP-2): a multicentre, double-blind, placebo-controlled, randomised trial. Lancet 2014;383:2232-7.

25 Vasileiou P, Tsioufis C, Lazaros G, Hadziyannis E, Kasiakogias A, Stefanadis $C$ et al. Interleukin-8 as a predictor of acute idiopathic pericarditis recurrences. A pilot study. Int J Cardiol 2014;172:e463-4.

26 Markel G, Imazio M, Koren-Morag N, Galore-Haskel G, Schachter J, Besser $\mathrm{M}$ et al. CEACAM1 and MICA as novel serum biomarkers in patients with acute and recurrent pericarditis. Oncotarget 2016;7:17885-95.

27 Antonopoulos AS, Oikonomou EK, Antoniades C, Tousoulis D. From the BMI paradox to the obesity paradox: the obesitymortality association in coronary heart disease. Obes Rev 2016;17:989-1000. 